\title{
Mechanisms of Action of Human Aldehyde Dehydrogenase Bright Cells in Therapy of Cardiovascular Diseases: Expression Analysis of Angiogenic Factors and Aldehyde Dehydrogenase Isozymes
}

Hannah Storrie White, Liisa Smith, Tracy Gentry and Andrew E. Balber*

Aldagen, Inc. 2810 Meridian Parkway Suite 148, Durham, NC 2771, USA

\begin{abstract}
Human stem cell populations that express high aldehyde dehydrogenase activity [ALDHbr cells] have angiogenic activity in preclinical models and have been used safely to treat patients in early clinical trials. Bone marrow ALDHbr cells are being developed for therapeutic use in ischemic cardiovascular diseases. The mechanisms by which ALDHbr cells repair ischemic tissue are unknown, but available data suggest that angiogenic factors released by ALDHbr cells are involved. Gene expression studies were performed, and bone marrow ALDHbr cells were found to express 69 of 84 angiogenic factors tested. The 25 most highly expressed genes included soluble cytokines and growth factors, cytokine receptors, extracellular matrix proteins, and cell-cell signaling receptors. ALDHdim bone marrow cells that do not express high levels of ALDH and that have no angiogenic activity in preclinical models expressed a different group of genes. CD105 and Ephrin B4 transcripts were expressed about 65-fold more highly in ALDHbr than ALDHdim cells, and expression of these proteins was demonstrated in ALDHbr cells by flow cytometry. Expression analysis probing all 19 ALDH isozymes and immunofluorescence both demonstrated that ALDH1A1, an enzyme that can generate retinoic acids from retinaldehyde, was the ALDH isozyme most highly over expressed in ALDHbr cells compared to ALDHdim cells. Transwell studies demonstrated that ALDHbr cells responded to hypoxic conditions and to factors released from human endothelial vein cells (HUVEC) with changes in expression of specific angiogenic factors. Soluble factors released from ALDHbr cells in transwell cultures under hypoxic conditions stabilized endothelial tubules formed by HUVEC. The results are consistent with the hypothesis that human bone marrow ALDHbr cells can promote angiogenesis in ischemic tissues of patients with cardiovascular disease by several mechanisms including the release of soluble mediators.
\end{abstract}

Keywords: Aldehyde dehydrogenase bright cells; Aldehyde dehydrogenase isozymes; Angiogenesis; Gene expression

\section{Introduction}

Restoration of blood flow to ischemic tissue may provide new treatment possibilities in cardiovascular disease. Various methods of inducing therapeutic angiogenesis, including treatment with recombinant angiogenic growth factor proteins and gene therapy constructs have been tested in clinical trials [1-5], but no technology is currently approved to drive angiogenesis for ischemic diseases in a clinical setting. Angiogenesis requires the orchestration of complex cellular and molecular events [6], and a single angiogenic factor, even delivered over a long period of time, may not be capable of inducing the full cascade of events necessary for restoration of tissue perfusion. Therapeutic cell populations, including bone marrow stem cells, have the potential to interact with ischemic tissues and deliver the variety of necessary factors in the correct spatial and temporal milieu, and several candidate cell products are in clinical development [7-13].

Cell populations with high aldehyde dehydrogenase (ALDH) enzymatic activity (ALDHbr cells) sorted from cord blood [14,15] and bone marrow $[16,17]$ are among the candidates therapies being investigated to treat cardiovascular diseases [18]. Autologous ALDHbr bone marrow cells have been used safely in clinical trials to treat adults with critical limb ischemia [19] and ischemic heart failure [20]. These trials provided clinical evidence for increased perfusion in the area of the damaged heart or leg injected with ALDHbr cells. Human ALDHbr cells home to sites of ischemic damage and have angiogenic activity in mouse models of hind limb ischemia [17], acute myocardial infarction [21], and pancreatic islet regeneration [22], and the restoration of tissue perfusion in the clinical studies may reflect this angiogenic activity.
The mechanisms of by which ALDHbr cells promote formation of new blood vessels in ischemic tissue are not known. ALDHbr cell populations are enriched in endothelial, mesenchymal, and hematopoietic progenitor cells that could play a direct role in forming new vessels $[16,17]$. However, these cells have not been observed to contribute significantly to newly formed vessels in vivo [16,21,22], suggesting that ALDHbr cells may secrete paracrine factors that regulate endothelial differentiation, division or survival under the conditions in ischemic tissue. Such factors could include extra-cellular matrix molecules that provide adhesion sites to endothelial cells, soluble chemokines and growth factors that promote endothelial cell migration, or other intercellular signaling molecules [6]. Retinoids are paracrine signaling molecules that are particularly interesting to consider in the context of ALDHbr cells, since these cells are defined by high ALDH activity and four of the 19 human ALDH isozymes generate retinoic acids from retinaldehydes [23]. Retinoids can have both pro- or anti-angiogenic effects by modulating endothelial cell

*Corresponding author: Andrew E. Balber, Cicada Biopharmaceutical Consulting 2608 Nation Ave., Durham NC 27707, USA, Tel: 919-943-6574; E-mail: andrew. balber@frontier.com

Received August 24, 2011; Accepted October 17, 2011; Published October 19, 2011

Citation: White HS, Smith L, Gentry T, Balber AE (2011) Mechanisms of Action of Human Aldehyde Dehydrogenase Bright Cells in Therapy of Cardiovascular Diseases: Expression Analysis of Angiogenic Factors and Aldehyde Dehydrogenase Isozymes. J Stem Cell Res Ther S1:001. doi:10.4172/2157-7633.S1-001

Copyright: ( 2011 White HS, et al. This is an open-access article distributed unde the terms of the Creative Commons Attribution License, which permits unrestricted use, distribution, and reproduction in any medium, provided the original author and source are credited. 
Citation: White HS, Smith L, Gentry T, Balber AE (2011) Mechanisms of Action of Human Aldehyde Dehydrogenase Bright Cells in Therapy of Cardiovascular Diseases: Expression Analysis of Angiogenic Factors and Aldehyde Dehydrogenase Isozymes. J Stem Cell Res Ther S1:001. doi:10.4172/2157-7633.S1-001

Page 2 of 9

cytokine and growth factors expression [24-33], migration [34-36], and vasculogenesis [37-40].

Identifying the specific paracrine angiogenic regulators expressed by ALDHbr cells may help elucidate the mechanisms by which ALDHbr cells promote angiogenesis. In the work presented here, gene expression arrays and flow cytometric and immunofluorescence analysis were used to study expression of angiogenic factors and ALDH isozymes by freshly isolated human bone marrow ALDHbr cells. How ALDHbr cells regulate expression of angiogenic factor genes when exposed to hypoxia and to hypoxic endothelial cells was also explored to identify angiogenic factors ALDHbr cells may express in ischemic tissue. Because experiments in the hind limb ischemia mouse model have suggested that the population of bone marrow mononuclear cells that do not over express ALDH (ALDHdim cells) can inhibit the angiogenic activity of ALDHbr cells [17], expression of angiogenic factors by ALDHbr and ALDHdim cells was compared. To explore the hypothesis that retinoids produced by ALDHbr cells might be among the paracrine factors that regulate angiogenesis, expression arrays were used to determine which ALDH human isozymes are over expressed in marrow and cord blood ALDHbr cells. Finally, an in vitro system was established to determine if paracrine factors released from ALDHbr cells can protect human endothelial cells from ischemic damage.

\section{Materials and Methods}

\section{ALDHbr cell preparation}

Bone marrow and cord blood units obtained from donors who had given informed consent under protocols approved by local institutional review boards were processed to remove red blood cells by the Sepax RM (Biosafe America, Inc., Houston, TX) automated density gradient protocol followed by lysing with ammonium chloride. Cells were then reacted with biodipy aminoacylacetaldehyde (BAAA), a fluorescent substrate for ALDH [14], and ALDHbr cells were isolated by fluorescence activated cell sorting [16]. Bone marrow mononuclear cells depleted of detectable ALDHbr cells by cell sorting (ALDHdim) were sorted from the same marrows and analyzed [16].

\section{Quantitative polymerase chain reaction [qRT-PCR]}

mRNA was isolated and purified from ALDHbr and ALDHdim cells using the Qiagen RNEasy Mini Plus kit. SABiosciences First Strand kit was used to generate cDNA templates and SYBR green based $\mathrm{RT}^{2}$ PCR arrays were used for gene array analysis. Arrays contained PCR controls and housekeeping gene controls and either 84 angiogenic gene factors or the 19 ALDH isozymes. All primer sequences are available at http://www.sabiosciences.com. The delta threshold cycle $(\Delta \mathrm{Ct})$ was compared between conditions to control for marrow to marrow variability, and the $\Delta \Delta \mathrm{Ct}$ method was used to determine relative gene expression between different cell populations. Genes with at least a four-fold difference in expression were considered differentially expressed. All data analysis was carried out using software provided online at http://www.sabiosciences.com/pcrarraydataanalysis.php.

\section{Flow cytometric analysis of cell surface antigens}

ALDHbr cells were stained with fluorescent antibodies to cell surface markers as previously described [16]. PE-Cy5 conjugated antiCD34 (BD Biosciences), PE conjugated anti-Ephrin receptor B4 (R\&D Systems), and APC conjugated anti-CD105 (BD Biosciences) were used. Flow cytometry was carried out using a FACS Calibur, and data analysis was carried out using CellQuest software.

\section{Transwell assays of release of paracrine factors by ALDHbr cells}

Human umbilical cord vein endothelial cells (HUVEC) (Lonza, Inc.,Gaithersburg, MD) were maintained in endothelial growth medium 2 (EGM2, Lonza) and used between passage 2 and 5. HUVECs were plated on thick Matrigel ${ }^{\mathbb{}}$ (BD Biosciences,) gels in a 24 well plate (70,000 cells/well) and tubules were allowed to form overnight as described by the manufacturer. ALDHbr cells $(50,000$ cells/well) were then added to a $0.4 \mu \mathrm{m}$ Transwell $^{\circledR}$ (Millipore, Inc.) inserted into the culture well. Transwell cultures were incubated under either normal or hypoxic, high $\mathrm{CO}_{2}$ conditions $\left(1 \% \mathrm{O}_{2}\right.$ and $\left.20 \% \mathrm{CO}_{2}\right)$ for 24 hours. Tubules were imaged before addition of ALDHbr cells and after 24 hour co-culture using an Olympus IX50 inverted microscope. Random fields from five replicates per condition were imaged and the study was repeated with four ALDHbr cell isolations (total number of branches counted $\geq 50$ per condition per study). The number of intact tubule branches was counted for each culture condition, and the ratio of tubule branches preserved under hypoxic conditions to tubule branches preserved under normoxic conditions was used to control for intrinsic tubule degradation.

\section{Immunofluorescence of ALDH1A1 expression}

Cytospin preparations of bone marrow ALDHbr and ALDHdim cells were reacted with monocolonal rabbit anti-human ALDH1Alantibody clone EP1933Y (Epitomics, Inc., Burlingame, CA) followed by a FITC-conjugated goat anti-rabbit IgG (Jackson ImmunoResearch Laboratories, West Grove, PA). Nuclei were counter-stained with DAPI. According to the manufacturer, EP1933Y recognizes an epitope between residues 325-350 of human ALDH1A1. BLAST searches revealed that human and rabbit ALDH1A1 sequences in this region differ only at residues 335-339 (human TPGVT vs. rabbit APEVN), and that only human ALDH1A2 and $1 \mathrm{~A} 3$ have significant homology to ALDH1A1 in this region.

\section{Results}

\section{Angiogenic gene expression profiling}

The expression of angiogenic genes by ALDHbr cells was determined by qRT-PCR using an array containing 84 angiogenic genes. Expression of 69 genes was detected $(\Delta \mathrm{Ct}<14)$; the other 15 genes were not expressed. The 25 most highly expressed genes $(\Delta \mathrm{Ct}<11)$ are listed in order of decreasing expression in Table 1 . The highly expressed genes, which include soluble growth factors and chemokines, cytokine receptors, intracellular signaling molecules and extra-cellular matrix (ECM) molecules, have diverse functions in angiogenesis, indicating that freshly isolated bone marrow ALDHbr cells express transcripts for a variety of different types of angiogenic factors.

\section{ALDH isozyme expression analysis}

A gene array of ALDH isozymes was used to determine whether ALDHbr cells over express the ALDH isozymes that generate retinoic acids that may modulate angiogenesis. Bone marrow and cord blood ALDHbr cells were studied, since both show angiogenic activity in preclinical animal models. Both cell populations expressed the same $11 \mathrm{ALDH}$ isozymes [ $\Delta \mathrm{Ct}<12$, ALDH1A1, $1 \mathrm{~B} 1,2,3 \mathrm{~B} 1,4 \mathrm{~A} 1,5 \mathrm{~A} 1,6 \mathrm{~A} 1$, 7A1, 9A1, 16A1, and 18A1] and did not express the same 8 isozymes $[\Delta \mathrm{Ct}>12, \mathrm{ALDH} 1 \mathrm{~A} 2,1 \mathrm{~A} 3,1 \mathrm{~L} 1,1 \mathrm{~L} 2,3 \mathrm{~A} 1,3 \mathrm{~B} 2,8 \mathrm{~A} 1]$. Although the relative expression of the isozymes in cord blood and bone marrow ALDHbr cells was very similar, ALDH4A1, ALDH9A1, and ALDH1B1 were significantly more highly expressed in bone marrow ALDHbr 
Citation: White HS, Smith L, Gentry T, Balber AE (2011) Mechanisms of Action of Human Aldehyde Dehydrogenase Bright Cells in Therapy of Cardiovascular Diseases: Expression Analysis of Angiogenic Factors and Aldehyde Dehydrogenase Isozymes. J Stem Cell Res Ther S1:001. doi:10.4172/2157-7633.S1-001

cells, and ALDH2 was more highly expressed in cord blood ALDHbr cells. Significantly, of the four isozymes that can oxidize retinaldehyde [ALDH 1A1, 1A2, 1A3, 8A1], only one isozyme, ALDH1A1, was expressed in ALDHbr cells, and it was more than 10-fold more highly expressed than ALDH9A1, the only other cytoplasmic ALDH isozyme expressed in ALDHbr cells.

\section{Differential gene profiles of ALDHbr and ALDHdim cells}

To identify the angiogenic factors that may contribute to the difference in angiogenic activity of ALDHbr and ALDHdim cells that has been observed in preclinical models, the differential expression of angiogenic factors was analyzed (Table 1). The top section of the Table compares the expression of the $25(\Delta \mathrm{Ct}>10)$ genes most highly expressed in ALDHbr cells to their expression in ALDHdim cells, and the bottom section of Table 1 includes genes that were weakly expressed by ALDHbr cells. Only genes showing a difference in expression between ALDHbr and ALDHdim cells that was greater than 4 -fold, or, if less than 4 -fold, was statistically significant $[\mathrm{p}<0.05]$ were included in the bottom section of Table 1. Expression of 43 genes [TGFA, TGFBR1, NRP1, ITGAV, CCL2, PLAU, EFNA3, HPSE, LAMA5, ANGPT2,

\begin{tabular}{|c|c|c|c|c|c|c|c|}
\hline \multirow[b]{2}{*}{ Symbol } & \multirow[b]{2}{*}{ Description } & \multicolumn{2}{|c|}{ ALDHbr } & \multicolumn{2}{|c|}{ ALDHdim } & \multicolumn{2}{|c|}{ ALDHbr vs. ALDHdim } \\
\hline & & mean $\Delta \mathbf{C t}$ & SD & mean $\Delta \mathbf{C t}$ & SD & Fold change & p value \\
\hline \multicolumn{8}{|c|}{ Most highly expressed genes in ALDHbr cells } \\
\hline ENG & CD105 [endoglin] & 4.31 & 0.95 & 10.38 & 1.44 & 66.93 & 0.04 \\
\hline TIMP1 & TIMP metallopeptidase inhibitor 1 & 4.78 & 0.23 & 5.26 & 0.11 & 1.39 & 0.03 \\
\hline IL8 & Interleukin 8 & 4.79 & 1.59 & 2.11 & 0.20 & -6.38 & $<0.001$ \\
\hline TGFB1 & Transforming growth factor, beta 1 & 5.39 & 0.52 & 4.05 & 0.59 & -2.54 & 0.03 \\
\hline EREG & Epiregulin & 5.41 & 0.90 & 6.11 & 0.27 & 1.63 & 0.20 \\
\hline EPHB4 & EPH receptor B4 & 5.94 & 0.59 & 11.96 & 0.23 & 64.60 & 0.01 \\
\hline AKT1 & V-akt murine thymoma viral oncogene homolog 1 & 6.02 & 0.76 & 7.10 & 0.13 & 2.11 & 0.10 \\
\hline HIF1A & Hypoxia inducible factor 1 , alpha subunit & 6.60 & 0.51 & 5.44 & 0.23 & -2.24 & 0.01 \\
\hline SPHK1 & Sphingosine kinase 1 & 7.06 & 0.78 & 10.28 & 0.22 & 9.30 & 0.01 \\
\hline COL18A1 & Collagen, type XVIII, alpha 1 & 7.30 & 0.91 & 10.76 & 0.73 & 10.97 & 0.03 \\
\hline VEGFA & Vascular endothelial growth factor $\mathrm{A}$ & 7.39 & 0.84 & 7.91 & 0.03 & 1.43 & 0.24 \\
\hline TIMP2 & TIMP metallopeptidase inhibitor 2 & 8.00 & 1.26 & 6.02 & 0.82 & -3.95 & 0.08 \\
\hline ANPEP & Alanyl (membrane) aminopeptidase & 8.36 & 1.85 & 7.21 & 1.00 & -2.23 & 0.28 \\
\hline S1PR1 & Sphingosine-1-phosphate receptor 1 & 8.53 & 0.39 & 4.81 & 0.70 & -13.15 & 0.01 \\
\hline FGFR3 & Fibroblast growth factor receptor 3 & 8.80 & 0.62 & 12.25 & 0.43 & 10.87 & 0.01 \\
\hline MMP2 & Matrix metallopeptidase 2 (Collagenase) & 8.83 & 1.43 & 13.31 & 0.27 & 22.44 & 0.07 \\
\hline MDK & Midkine (neurite growth-promoting factor 2) & 9.03 & 1.21 & 11.13 & 0.64 & 4.28 & 0.11 \\
\hline MMP9 & Matrix metallopeptidase 9 (Collagenase) & 9.18 & 0.92 & 2.44 & 0.94 & -106.64 & 0.02 \\
\hline ID1 & Inhibitor of DNA binding 1 & 9.33 & 0.39 & 8.49 & 0.33 & -1.79 & 0.02 \\
\hline PTGS1 & Prostaglandin-endoperoxide synthase 1 & 9.40 & 0.54 & 12.21 & 0.74 & 7.01 & 0.02 \\
\hline PROK2 & Prokineticin 2 & 9.45 & 2.37 & 7.44 & 1.44 & -4.02 & 0.33 \\
\hline TYMP & Thymidine phosphorylase & 9.50 & 0.38 & 6.62 & 0.67 & -7.34 & 0.01 \\
\hline SERPINF1 & EDG1 & 9.61 & 0.77 & 9.86 & 0.56 & 1.19 & 0.55 \\
\hline IL1B & Interleukin 1 , beta & 9.63 & 1.44 & 6.83 & 0.46 & -6.97 & $<0.001$ \\
\hline PECAM1 & CD31 & 9.69 & 2.57 & 7.90 & 6.91 & -3.45 & 0.29 \\
\hline \multicolumn{8}{|c|}{ Other genes differentially expressed in ALDbr vs. ALDHdim cells } \\
\hline TEK & TEK tyrosine kinase, endothelial & 10.57 & 1.12 & 13.32 & 0.52 & 6.75 & 0.14 \\
\hline TNFAIP2 & Tumor necrosis factor, alpha-induced protein 2 & 10.78 & 0.62 & 8.53 & 0.19 & -4.77 & $<0.001$ \\
\hline CXCL1 & Chemokine (C-X-C motif) ligand 1 & 10.82 & 1.77 & 7.64 & 1.42 & -9.10 & 0.11 \\
\hline THBS1 & Thrombospondin 1 & 10.97 & 1.69 & 8.24 & 1.18 & -6.64 & 0.05 \\
\hline ID3 & Inhibitor of DNA binding 3 & 10.99 & 0.49 & 7.51 & 0.16 & -11.14 & $<0.001$ \\
\hline ANGPT1 & Angiopoietin 1 & 11.15 & 0.81 & 13.94 & 0.90 & 6.96 & 0.03 \\
\hline TNF & Tumor necrosis factor & 11.76 & 1.22 & 6.17 & 0.74 & -48.27 & 0.01 \\
\hline EFNB2 & Ephrin-B2 & 12.66 & 0.95 & 11.10 & 0.41 & -2.94 & 0.03 \\
\hline VEGFC & Vascular endothelial growth factor C & 12.99 & 1.96 & 10.19 & 5.73 & -6.96 & 0.29 \\
\hline IL6 & Interleukin 6 (interferon, beta 2) & 13.96 & 0.45 & 9.22 & 0.96 & -26.86 & 0.04 \\
\hline IFNG & Interferon, gamma & 13.99 & 0.92 & 7.66 & 0.89 & -80.49 & 0.01 \\
\hline PLXDC1 & Plexin domain containing 1 & 14.00 & 1.12 & 11.23 & 1.77 & -6.83 & 0.13 \\
\hline CXCL6 & Chemokine (C-X-C motif) ligand 6 & 14.69 & 0.70 & 10.38 & 1.08 & -19.87 & 0.05 \\
\hline KDR & Kinase insert domain receptor/VEGF receptor & 15.08 & 1.22 & 13.24 & 0.61 & -3.60 & 0.03 \\
\hline COL4A3 & Collagen, type IV, alpha 3 & 15.19 & 1.22 & 13.55 & 0.44 & -3.10 & 0.04 \\
\hline HAND2 & Heart and neural crest derivatives expressed 2 & 15.19 & 1.22 & 13.30 & 0.54 & -3.70 & 0.02 \\
\hline
\end{tabular}

Table 1: Expression of angiogenic genes by ALDHbr and ALDHdim cell populations of human bone marrow cells. Top section. QRT-PCR based expression data for the 25 gene products most highly expressed in ALDHbr cells are listed in order from the most to least abundant transcript. The Fold Change columns shows the fold up-regulation [positive numbers, red] or down regulation [negative numbers, blue] of expression of ALDHbr cells compared to ALDHdim cells. The last column indicates the $p$-value [t-test] for the fold regulation of expression between the populations. Bottom Section. Expression data for other genes selected as described in the text. 
Citation: White HS, Smith L, Gentry T, Balber AE (2011) Mechanisms of Action of Human Aldehyde Dehydrogenase Bright Cells in Therapy of Cardiovascular Diseases: Expression Analysis of Angiogenic Factors and Aldehyde Dehydrogenase Isozymes. J Stem Cell Res Ther S1:001. doi:10.4172/2157-7633.S1-001

FIGF, NRP2, ANGPTL3, ANGPTL4, ITGB3, THBS2, IGF1, CXCL9, CXCL10, LECT1, PDGFA, EGF, CXCL5, FLT1, IFNB1, BAI1, FGF2, PGF, SERPINF1, PF4, CXCL3, HGF, JAG1, STAB1, NOTCH4, CDH5, CCL11, FGF1, IFNA1, LEP, PLG, TGFB2, TIMP3] did not meet these criteria. CD105 and EPHB4 were the most upregulated genes in ALDHbr cells. The next 10 most upregulated genes in ALDHbr cells included cytokines, matrix modifying proteases, and intracellular signaling molecules. The most down regulated gene in ALDHbr cells compared to ALDHdim cells was the MMP9 protease; interleukins, TNF, and inflammatory cytokines and chemokines were among the most downregulated genes.

Differential expression of ALDH isozymes by ALDHbr than ALDHdim cells was also studied (Table 2). Eight isozymes (ALDH1A1, $1 \mathrm{~B} 1,4 \mathrm{~A} 1,5 \mathrm{~A} 1,18 \mathrm{~A} 1,6 \mathrm{~A} 1,9 \mathrm{~A} 1$, and 16A1) were upregulated in ALDHbr cells, and ten isozymes showed no difference in expression. Only ALDH2, the most highly expressed ALDH isozyme in ALDHdim cells, had a lower $\Delta \mathrm{Ct}$ in ALDHdim cells $(\Delta \mathrm{Ct}=5.00)$ than ALDHbr cells $(\Delta \mathrm{Ct}=6.65)$, but this $\Delta \mathrm{Ct}$ value was significantly higher than the $\Delta \mathrm{Ct}$ value for the most highly expressed ALDHbr isozyme (ALDH1A1, $\Delta \mathrm{Ct}=1.89$ ), and ALDH2 was only 3.79 fold upregulated in ALDHdim cells. Transcripts for ALDH1A1 were 102-fold more abundant in ALDHbr cells than ALDHdim cells.

\section{Expression of angiogenic proteins by ALDHbr cells}

Flow cytometry and immunofluorescence were used to examine expression of proteins corresponding to abundant transcripts in ALDHbr cells (Figure 1). CD105 and EphB4 were the most highly upregulated genes in ALDHbr cells, and flow analysis demonstrated that $44.7 \pm 14.1 \%$ of ALDHbr cells expressed CD105 and $41.8 \pm 13.8$ $\%$ expressed EphB4 (Figure 1A-C). Multicolor flow studies were performed to analyze co-expression of CD105, EphB4, and CD34 in ALDHbr cells. These analyses showed that $88.2 \pm 11.1 \%$ of ALDHbr cells expressing either EphB4 or CD105 co-expressed the other marker (Figure 1D), but only $22.0 \pm 10.1 \%$ of ALDHbr/EphB4 ${ }^{+}$cells and 24.6 $\pm 13.9 \%$ of $\mathrm{ALDHbr} / \mathrm{CD} 105^{+}$cells expressed CD34 (Figure $1 \mathrm{E}, \mathrm{F}$ ). Immunofluorescence analysis was used to confirm that ALDH1A1 protein, like its transcript, was expressed in ALDHbr cells. Given the characteristics of the EP1933Y monoclonal antibody presented in Materials and Methods and the observation that neitherALDH1A2 or ALDH1A3 transcripts were detected in ALDHbr or ALDHdim bone marrow cells (Table 2), EP1933Y is a highly specific reagent to detect ALDH1A1 expression in these cell populations. Intense cytoplasmic staining was observed in ALDHbr cells incubated in this antibody, while no staining was observed in ALDHdim cells (Figure 2), indicating that ALDHbr cells express higher levels of ALDH1A1 than ALDHdim cells.

Interactions between ALDHbr cells and endothelial cells in hypoxic cultures

An in vitro model was established to determine if paracrine factors

\begin{tabular}{|c|c|c|c|c|c|}
\hline ALDH isozyme & $\begin{array}{l}\text { UCB ALDHbr } \\
\text { Mean } \Delta \text { Ct (SD) }\end{array}$ & $\begin{array}{c}\text { BM ALDHbr } \\
\text { Mean } \Delta \text { Ct }(\text { SD) }\end{array}$ & $\begin{array}{l}\text { BM ALDHdim } \\
\text { Mean } \Delta \text { Ct (SD) }\end{array}$ & $\mathrm{BM}$ Fold regulation ${ }^{\mathrm{b}}$ & Compartment $^{c}$ \\
\hline $1 \mathrm{~A} 1$ & $3.36(0.63)$ & $1.90(0.73)$ & $8.32(0.65) \#$ & 102.96 & Cytoplasmic \\
\hline 1B1 & $10.00(0.55)^{\star}$ & $7.40(0.79)$ & $11.28(0.66) \#$ & 13.35 & Mitochondrial \\
\hline $4 \mathrm{~A} 1$ & $7.15(0.49)^{*}$ & $4.79(0.70)$ & 8.07 (1.01)\# & 10.38 & Mitochondrial \\
\hline $5 \mathrm{~A} 1$ & $6.75(0.16)$ & $5.48(0.71)$ & $8.28(0.90) \#$ & 8.11 & Mitochondrial \\
\hline $18 \mathrm{~A} 1$ & $5.16(0.41)$ & $4.07(0.61)$ & 7.08 (0.66)\# & 7.51 & Mitochondrial \\
\hline $6 \mathrm{~A} 1$ & $5.68(0.42)$ & $5.07(0.62)$ & $7.04(0.86) \#$ & 4.37 & Mitochondrial \\
\hline $9 A 1$ & $7.36(0.34)^{\star}$ & $5.47(0.68)$ & 7.46 (0.91)\# & 4.27 & Cytoplasmic \\
\hline $16 \mathrm{~A} 1$ & $6.73(0.40)$ & $5.53(0.78)$ & 7.63 (0.55)\# & 4.26 & Unknown \\
\hline $7 \mathrm{~A} 1$ & $7.10(0.52)$ & $7.22(0.25)$ & 8.54 (0.99)\# & 2.56 & Cytoplasmic \\
\hline $3 \mathrm{~A} 2$ & $7.76(0.25)$ & $7.22(0.61)$ & $7.95(0.48)$ & 1.5 & Microsomal \\
\hline $1 \mathrm{~A} 3$ & $12.84(1.54)$ & $12.38(1.23)$ & $12.37(1.01)$ & -1.24 & Cytoplasmic \\
\hline 3B1 & $7.06(1.13)$ & $6.20(0.52)$ & $5.53(0.83)$ & -1.29 & Cytoplasmic \\
\hline 2 & $4.73(0.53)^{*}$ & $6.65(0.54)$ & $5.00(0.60) \#$ & -3.79 & Mitochondrial \\
\hline $1 \mathrm{~L} 1$ & $13.92(1.20)$ & $13.42(0.92)$ & $13.20(1.03)$ & -1.18 & Cytoplasmic \\
\hline $8 \mathrm{~A} 1$ & $13.70(0.77)$ & $13.49(0.75)$ & $13.12(1.03)$ & -1.37 & Cytoplasmic \\
\hline $1 \mathrm{~L} 2$ & $12.92(0.64)$ & $13.28(1.06)$ & $11.84(0.75)$ & -1.44 & Mitochondrial \\
\hline $1 \mathrm{~A} 2$ & $15.23(1.33)$ & $13.60(0.83)$ & $13.12(1.03)$ & -1.51 & Cytoplasmic \\
\hline 3B2 & $13.63(0.84)$ & $13.60(0.83)$ & $13.12(1.03)$ & -1.51 & Cytoplasmic \\
\hline $3 \mathrm{~A} 1$ & $13.67(1.93)$ & $13.34(0.97)$ & $12.99(1.00)$ & -2.34 & Cytoplasmic \\
\hline
\end{tabular}

a $\quad \mathrm{N}=10$ for BM ALDH ${ }^{\text {br }} ; \mathrm{n}=5$ for BM ALDH ${ }^{\text {dim; }} \mathrm{N}=4$ for UCB ALDH ${ }^{\text {br }}$.

b Positive fold-regulation, ALDHbr>ALDHdim; Negative, ALDHdim>ALDHbr.

c Subcellular localization according to reference 22

$p<0.05$ for UCB ALDHbr as compared to bone marrow ALDHbr; t-test

\# $\quad \mathrm{p}<0.05$ for bone marrow ALDHbr as compared to bone marrow ALDHdim; $\mathrm{N}=5$ matched pairs, t-test

Table 2: Quantitative reverse transcriptase polymerase chain reaction analysis of 19 ALDH isozyme transcripts in ALDHbr cells from cord blood and of ALDHbr and ALDHdim cells from bone marrow. 
Citation: White HS, Smith L, Gentry T, Balber AE (2011) Mechanisms of Action of Human Aldehyde Dehydrogenase Bright Cells in Therapy of Cardiovascular Diseases: Expression Analysis of Angiogenic Factors and Aldehyde Dehydrogenase Isozymes. J Stem Cell Res Ther S1:001. doi:10.4172/2157-7633.S1-001
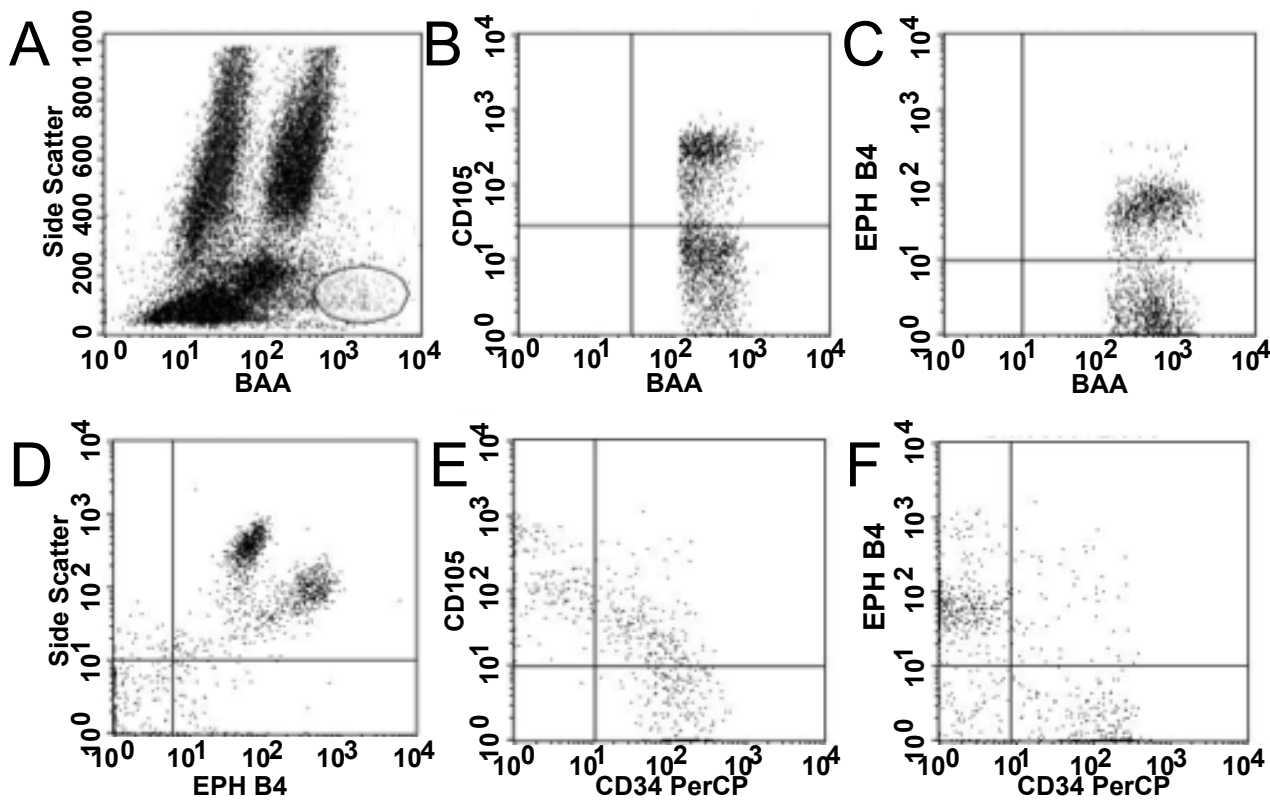

Figure 1: Mutliparameter flow cytometric analysis of expression of surface antigens endoglin/CD105, EphB4, and CD34 by bone marrow ALDHbr cells (A) ALDHbr cells defined on the basis of high BAAA fluorescence and low side scatter are shown in the oval gate. (B) and (C) show expression of CD105 and EphB4 respectively, and (D) shows co-expression of CD105 and Eph B4 by CD105+ or EphB4+ cells in the ALDHbr gate shown in (A). CD105 and Eph B4 are predominantly expressed on ALDH ${ }^{\mathrm{b} r} / \mathrm{CD} 34$ - cells (E, F, respectively).

released from ALDHbr cells stabilize interactions among endothelial cells in tubular networks and between endothelial tubules and the extracellular matrix under hypoxic conditions. HUVEC tubules formed under normoxic conditions on Matrigel were exposed to hypoxia either in the presence or absence of ALDHbr cells placed in a transwell insert, and the persistence of the branched network of HUVEC was measured by counting branches arising from individual endothelial cells. Figure 3

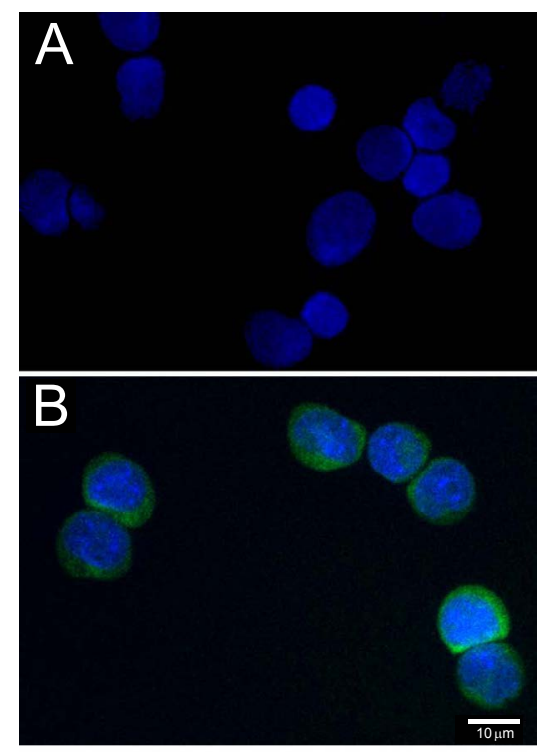

Figure 2: Immunofluorescence analysis of ALDH1A1 expression. Cytospin preparations of $A L D H d i m(A)$ and $A L D H b r(B)$ cells incubated with rabbit monoclonal antibody EP1933Y to ALDH1Al and FITC conjugated goat antirabbit IgG. Nuclei are counterstained with DAPI. Original magnification $400 \mathrm{x}$. shows a representative areas of plates at the time endothelial networks were transferred to hypoxic conditions and after 24 hours in transwell cultures with and without ALDHbr cells. Treating HUVEC networks with ALDHbr cells under normoxic conditions had no effect on network persistence; the number of branches in HUVEC networks maintained under normoxic conditions in co-cultures with ALDHbr cells for $24 \mathrm{~h}$ was $98 \pm 4 \%$ (mean $+/$ - SD) of the initial value $(n=4)$. In contrast, when HUVEC tubules were held under hypoxic conditions for $24 \mathrm{~h}$, tubules retracted and branching decreased to $79 \pm 16 \%$ of the initial value. Hypoxic HUVEC tubules cultured in transwells with ALDHbr cells maintained branched network morphology and the number of intact branches increased to $127 \pm 13 \%$ of the control value. The difference in tubule branching between hypoxic HUVEC cultured in transwells with and without ALDHbr cells was statistically significant $(\mathrm{p}<0.05, \mathrm{n}=4)$.

To identify paracrine factors that may be released by ALDHbr cells to stabilize HUVEC networks in this model system, and, by extension, factors that may stabilize endothelium in ischemic tissue in vivo, changes in expression of angiogenic transcripts in ALDHbr cells cultured under hypoxic conditions with and without HUVEC were measured. Expression of 49 of the 84 genes in the angiogenic array was upregulated, and 9 were down regulated greater than 2 -fold when ALDHbr cells were cultured under hypoxic conditions. Difference in expression of the 20 genes shown in Table 3 reached statistical significance $(\mathrm{p}<0.05, \mathrm{n}=4)$. These transcripts included transcriptions factors (HAND2, ID1), cell signaling molecules (EFNA1, S1PR1), soluble chemokines and cytokines (CXCL5, CXCL6, CXCL9, FGF1, IL1B, LEP), VEGF receptor 2, and ECM molecules (PLAU, PLG, TIMP2, TMP3, COLL4A3). Only expression of collagen 18A1, was significantly down regulated by hypoxia. When ALDHbr cells were cultured in transwells with HUVECs under hypoxic conditions, 56 transcripts were upregulated, and none were down regulated, more than 2 -fold. Differences in expression of 20 of the angiogenic genes upregulated in 
Citation: White HS, Smith L, Gentry T, Balber AE (2011) Mechanisms of Action of Human Aldehyde Dehydrogenase Bright Cells in Therapy of Cardiovascular Diseases: Expression Analysis of Angiogenic Factors and Aldehyde Dehydrogenase Isozymes. J Stem Cell Res Ther S1:001. doi:10.4172/2157-7633.S1-001

Page 6 of 9

hypoxic transwell cocultures were statistical significant $(\mathrm{p}<0.05 ; \mathrm{n}=3$; Table 3). Table 3 shows that 12 of these upregulated transcripts were also upregulated in ALDHbr cells cultured under hypoxia without HUVEC cells. Expression of nine transcripts in hypoxic ALDHbr cells increased further, of 2 transcripts decreased, and of one transcript did not change significantly in response to HUVEC (Table 3). In addition, hypoxic ALDHbr cells specifically and significantly $(\mathrm{p}<0.05)$ upregulated expression of 8 transcripts shown in Table 3 in response to the presence of HUVEC cells in transwell cultures.

\section{Discussion}

The data presented above represent the first analysis of expression of angiogenic factors by ALDHbr cells. Expression of 69 of the 84 angiogenic factors in the microarray was detected. Soluble angiogenic molecules that can be secreted from ALDHbr cells and act in a paracrine fashion are prominent among the 25 most highly expressed genes. These include soluble cytokines (IL8, TGF $\beta$, VEGF, and MDK), soluble molecules that can alter the structure of the extracellular matrix (TIMP1, COL18A, TIMP2, ANPEP, MMP2), and enzymes that generate soluble molecules that are angiogenic signals to endothelial cells (SPHK1, PTGS1). Retinoids may also be among the soluble angiogenic mediators produced by ALDHbr cells. ALDH1A1 is the
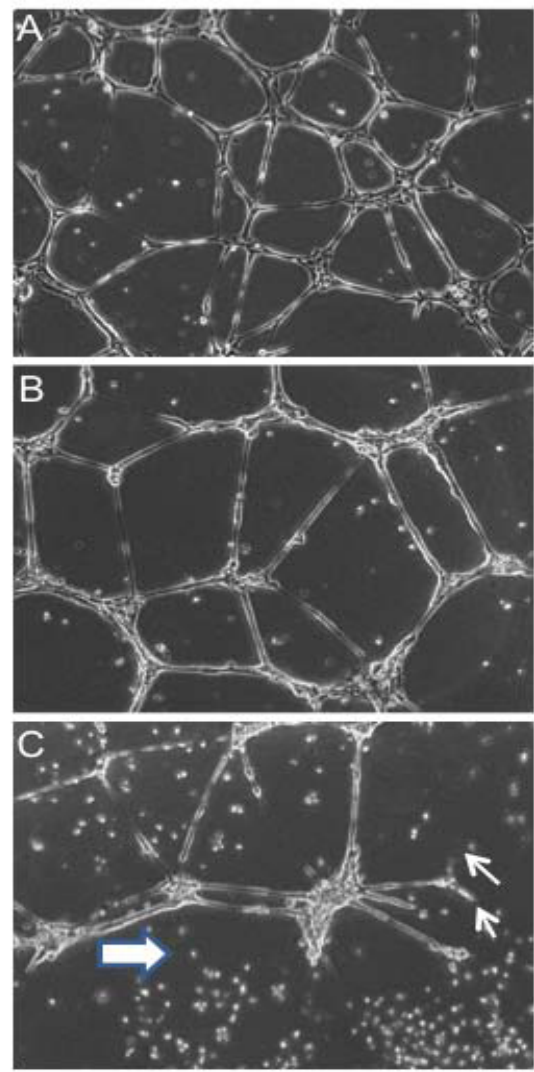

Figure 3: Effect of hypoxia on HUVEC tubular network maintained with and without ALDHbr cells in transwell cultures. (A)HUVEC cells plated on extracellular matrix in the bottom of transwell culture plates were allowed to form tubular networks under normoxic conditions. Transwell cultures were then incubated under hypoxic conditions for $24 \mathrm{~h}$ either with (B) or without (C) ALDHbr cells added to the top chamber of the transwell cultures. HUVEC maintained defined branching structures in transwells with ALDHbr cells (B), while tubules without ALDHbr cells $(C)$ retract (thin arrows) and areas of cell necrosis were present (wide arrow)

\begin{tabular}{|l|c|c|c|c|}
\hline & \multicolumn{2}{|c|}{$\begin{array}{c}\text { ALDHbr vs. Hypoxic } \\
\text { ALDHbr in monoculture }\end{array}$} & $\begin{array}{r}\text { ALDHbr vs. Hypoxic ALDHbr with } \\
\text { HUVEC in transwells }\end{array}$ \\
\hline Gene & $\begin{array}{c}\text { Fold } \\
\text { Regulation }\end{array}$ & p value & Fold Regulation & p value \\
\hline
\end{tabular}

Genes significantly upregulated under both culture conditions

\begin{tabular}{|l|c|c|c|c|}
\hline CXCL5 & 16.0 & $<0.001$ & 140.3 & 0.02 \\
\hline EFNA1 & 11.2 & $<0.01$ & 7.2 & 0.04 \\
\hline CXCL6 & 11.0 & 0.01 & 11.6 & 0.03 \\
\hline TIMP3 & 10.0 & $<0.01$ & 20.3 & 0.02 \\
\hline COL4A3 & 9.1 & $<0.01$ & 15.6 & 0.02 \\
\hline PLAU & 9.1 & 0.03 & 6.0 & 0.01 \\
\hline FGF1 & 6.7 & $<0.001$ & 22.6 & 0.02 \\
\hline PLG & 6.7 & $<0.001$ & 24.3 & 0.02 \\
\hline LEP & 6.4 & $<0.001$ & 23.7 & 0.02 \\
\hline CCL11 & 5.8 & $<0.001$ & 22.2 & 0.02 \\
\hline HAND2 & 5.6 & $<0.001$ & 21.9 & 0.02 \\
\hline PGF & 3.6 & 0.0376 & 18.5 & 0.01 \\
\hline
\end{tabular}

Genes significantly regulated in hypoxic monoculture only

\begin{tabular}{|l|c|l|l|l|}
\hline IL1B & 6.4 & 0.03 & & \\
\hline KDR & 4.7 & 0.04 & & \\
\hline TIMP2 & 4.2 & 0.03 & & \\
\hline CXCL9 & 3.7 & 0.01 & & \\
\hline JAG1 & 3.3 & 0.01 & & \\
\hline S1PR1 & 3.1 & 0.04 & & \\
\hline ID1 & 1.8 & 0.02 & & \\
\hline COL18A1 & -9.2 & 0.03 & & \\
\hline
\end{tabular}

Genes significantly upregulated in hypoxic cultures with HUVEC only

\begin{tabular}{|l|c|c|c|}
\hline IFNA1 & & 16.1 & 0.02 \\
\hline EFNA3 & & 11.9 & 0.03 \\
\hline IL6 & & 9.8 & 0.02 \\
\hline ANGPTL3 & & 8.1 & 0.02 \\
\hline CCL2 & & 7.7 & 0.04 \\
\hline EGF & & 6.4 & 0.04 \\
\hline THBS2 & & 3.5 & 0.03 \\
\hline SPHK1 & & & $<0.01$ \\
\hline
\end{tabular}

Table 3: Expression of angiogenesis genes by freshly isolated ALDHbr cells and ALDHbr cells cultured in transwells under hypoxic conditions with and without HUVEC networks. Cultures were maintained as described in the text. Values are fold change in mean expression of genes in the angiogenic array that were significantly $(p<0.05)$ upregulated (positive values) or down regulated (negative values). Gene expression was measured by QRT-PCR.

most highly expressed ALDH superfamily gene in human ALDHbr bone marrow and cord blood ALDHbr cell populations, and ALDH1A1 transcripts are more than 102 times more abundant in ALDHbr cells than ALDHdim cells. Immunofluorescence studies showed intense cytoplasmic staining with anti-ALDH1A1, demonstrating that the ALDH1A1 protein is also highly expressed in ALDHbr cells. None of the other ALDH isozymes that generate retinoic acids were expressed by these cells. Expression of only one ALDH1 subfamily isozyme 
Citation: White HS, Smith L, Gentry T, Balber AE (2011) Mechanisms of Action of Human Aldehyde Dehydrogenase Bright Cells in Therapy of Cardiovascular Diseases: Expression Analysis of Angiogenic Factors and Aldehyde Dehydrogenase Isozymes. J Stem Cell Res Ther S1:001. doi:10.4172/2157-7633.S1-001

that can oxidize retinoids appears to be a general characteristic of ALDHbr cells isolated by sorting with the BAAA substrate [18]. Retinoid oxidizing ALDH isozymes have a large substrate passage that permits bulky aldehyde substrates like retinoids and BAAA access to the catalytic site, while isozymes that oxidize small metabolic aldehyde substrates exclude such bulky substrates [41]. Thus, using the ALDH substrate BAAA may select for cells expressing high levels of enzymes that can generate retinoid signaling molecules. Both the angiogenic and ALDH microarray results, then, are consistent with the idea that ALDHbr cells can provide a variety of soluble angiogenic signaling molecules to endothelial and identify the specific gene products that are likely to participate.

Analysis of the branching of HUVEC tubular networks in hypoxic co-culture with ALDHbr cells demonstrated that soluble molecules released by ALDHbr cells exposed to hypoxic endothelium are active in stabilizing endothelial cell interactions under hypoxia. Adding ALDHbr cells to the upper chamber of the transwells allowed the endothelial networks to maintain their branching structure as compared to controls with no source of angiogenic factors. In this model, paracrine factors may influence interactions among HUVEC cells as well as their interactions with the matrix. The branching assay was adopted to model the effects of paracrine factors on established endothelial interactions in the tissues of chronic ischemia patients where existing endothelial interactions with other endothelial cells, the matrix, and other cell types must be maintained while damaged cells are replaced, angiogenesis occurs, and new interactions are formed [6].

Co-culture of ALDHbr cells with HUVECs in transwells also provided direct evidence that ALDHbr cells change expression of angiogenic factors in response to soluble factors released from endothelial cells under hypoxic conditions. These soluble endothelial cell factors further upregulated expression by ALDHbr cells of 12 of the genes upregulated by hypoxia alone and significantly upregulated expression of an additional 8 genes compared to freshly isolated cells alone. The upregulated genes encode soluble cytokines and growth factors, enzymes that control modifications of the matrix, angiogenic transcription factors, and receptors for angiogenic cytokines. Thus, the results of expression analysis in hypoxic transwell co-cultures confirm that ALDHbr cells specifically respond to soluble signals from endothelial cells by producing additional signals that can regulate the activities of the endothelial cells.

Several genes encoding soluble angiogenic factors were also expressed by ALDHdim cells. Nevertheless, ALDHdim cells have less angiogenic activity in culture and preclinical animal models than ALDHbr cells $[16,17,21,22]$. This suggests that the angiogenic cytokines that are differentially expressed in ALDHbr cells and/or the products of ALDH1A1 are particularly important in mediating angiogenic activity in the ischemic environment. In addition, several of the genes that were most highly expressed by ALDHdim compared to ALDHbr cells were soluble inflammatory cytokines INFG, TNF, and IL6 that can have anti-angiogenic activity and impede repair of cardiovascular tissue [42]. Because ALDHbr cells are rare (approximately $1 \%$ of the marrow mononuclear cells), this may explain why co-infusing ALDHdim cells with ALDHbr cells inhibits the angiogenic activity of ALDHbr cells in the hind limb ischemia model [17].

We studied expression of angiogenic factors by ALDHbr cells maintained in culture under hypoxic conditions to determine what angiogenic factors these cells might express when exposed to ischemic tissue. Expression of one gene in the angiogenic array, COL18A, was down regulated, expression of 19 genes was upregulated, and expression of the other 49 angiogenic genes expressed by freshly isolated ALDHbr cells was not significantly changed in hypoxic cultures. These results show, first, that ALDHbr continue to express many angiogenic gene products under hypoxic conditions and, second, that these cells regulate the expression of a specific subset of angiogenic conditions under hypoxia. An interesting aspect of the response of ALDHbr cells to hypoxia is that several angiogenic cytokines (CD105, HIFa, AKT, and VEGFA, for example) that are generally upregulated by hypoxia [6], were highly expressed by freshly isolated ALDHbr cells, and expression of these factors was not significantly changed when ALDHbr cells were exposed to hypoxia. The stem cell niche in bone marrow is thought to be hypoxic [43], and expression of these cytokines in ALDHbr cells isolated from marrow may reflect residence in that environment. Hypoxia also alters cell metabolism, and the high expression of mitochondrial ALDH4A1 and 2A in freshly isolated ALDHbr cells may also be a result of their exposure to the niche. Compared to ALDHdim cells, ALDHbr cells over expressed transcripts for five mitochondrial and three cytoplasmic ALDH isozymes that oxidize aldehydes formed during intermediary carbohydrate metabolism and lipid oxidation [23]. Other workers have also found that ALDHbr cell populations express mitochondrial isozymes ALDH2 [44] and ALDH4A1 [45]. Upregulation of mitochondrial ALDH isozymes may reflect the metabolic demands of the hypoxic niche; mitochondrial biogenesis is an integral part of the differentiation of hematopoietic stem cells from pluripotent progenitors in the stem cell niche [46,47]. Adaptation to low oxygen environments may augment the ability of ALDHbr cells to function in ischemic tissues of patients.

The similarity of the ALDH isozyme expression profiles of ALDHbr cells isolated from human bone marrow and cord blood cells is remarkable given that these cell populations differ significantly in the percentage of cells that express several canonical stem cell surface antigens such as CD34 and CD133 [14-17]. Several workers have now reported that ALDHbr cells have higher stem cell activity than ALDHdim cells bearing similar surface antigens associated with stem cell activity [18]. Retinoid mediated regulatory changes have recently been shown to alter the ability of $\mathrm{CD} 34^{+}$cells in the peripheral circulation of coronary artery disease patients to home to ischemic tissue [48], a finding consistent with the idea that retinoids produced by ALDH play an important role in controlling the ability of stem cells to respond to ischemic damage.

Our gene expression studies also suggest that ALDHbr cells can participate in angiogenesis by mechanisms in addition to production of soluble mediators. First, the 25 most highly expressed genes include regulatory proteins (EREG, AKT, HIF1A, and ID1) that regulate multiple angiogenic pathways. Second, receptors for angiogenic molecules including CD105, S1PR1, and FGFR3 were also highly expressed by ALDHbr cells. CD105 was the most highly expressed gene in the array and was expressed about 66-fold more highly by ALDHbr cells than ALDHdim cells. CD105 is a multi-functional non-signaling receptor that modulates binding of TGF $\beta$-activating-BMP family cytokines, regulates cell adhesion and migration, modulates angiogenic cytokine secretion and has a variety of other functions [49-51]. The function of CD105 in ALDHbr cells remains to be determined. Flow cytometry showed that CD105 was expressed primarily by the CD34 subpopulation of ALDHbr cells. Expression of CD105, S1PR1, and FGFR3 suggests that ALDHbr can interact with other cells, including endothelial cells, in cross-signaling. The transwell studies already discussed provide direct evidence that ALDHbr cells respond to signals 
Citation: White HS, Smith L, Gentry T, Balber AE (2011) Mechanisms of Action of Human Aldehyde Dehydrogenase Bright Cells in Therapy of Cardiovascular Diseases: Expression Analysis of Angiogenic Factors and Aldehyde Dehydrogenase Isozymes. J Stem Cell Res Ther S1:001. doi:10.4172/2157-7633.S1-001

produced by HUVEC under hypoxia. Finally, the high expression of EphB4 suggests that ALDHbr cells may interact with endothelial cells and also regulate angiogenesis by an additional mechanism, direct cell to cell contact [51-53]. Interaction of ephrin receptors on one cell with cognate ephrin ligands on another can initiate angiogenic activity in both cells. Ephrin receptor B4 was the sixth most highly expressed gene in the array and, like CD105, was expressed about 66-fold more strongly in ALDHbr than ALDHdim cells. Flow cytometry showed that EphB4 was almost always co-expressed with CD105 in ALDHbr cells. Genes for ephrin ligands EFNA1 and A2 were weakly expressed by freshly isolated cells ALDHbr cells, but were upregulated about tenfold in response to hypoxia and HUVEC in culture, suggesting that ALDHbr cells can participate in a variety of cell interactions through the ephrin system. Expression of any member of the ephrin signaling system by ALDHbr cells has not been previously reported.

These studies document the broad angiogenic potential of ALDHbr cells from human bone marrow. These cells expressed angiogenic genes encoding soluble growth factors, regulatory molecules, and ECM molecules, and soluble factors released from these protected endothelial tubules from damage from hypoxia. In addition, ALDHbr cells engaged in cross-signaling with endothelial cells, responding to soluble molecules produced by endothelial cells by changing expression of a specific set of genes encoding angiogenic factors. Gene expression studies showed that ALDHbr cells strongly expressed genes for several receptors of cytokines produced by endothelial cells. Furthermore, these studies showed that such paracrine signaling is not the only possible mechanism by which ALDHbr cells may interact with endothelium in the ischemic tissues, for ALDHbr cells strongly expressed molecules involved in ephrin signaling. The angiogenic factors that are differentially expressed in ALDHbr cells and ALDHdim cells and that are upregulated in response to hypoxia and soluble products from endothelial are candidates for further work detailing the multiple mechanisms by which ALDHbr cells participate in formation of new blood vessels, increase tissue perfusion, and repair ischemic damage in patients with cardiovascular disease.

\section{Disclosures of Interest}

HSW is an employee of Aldagen, Inc., a company that is developing therapeutic uses for ALDHbr cells. TG and AEB hold equity or options in Aldagen, and $\mathrm{AEB}$ consults for the company.

\section{Acknowledgements}

We are grateful to Nick Herman and Brittany Phillips for preparing cells, to Darren Hickerson, Alan Nguyen, Elizabeth Nieman, and Hank Saye for flow sorting, and to Joanne Kurtzberg, MD for providing materials.

\section{References}

1. Rajagopalan S, Olin J, Deitcher S, Pieczek A, Laird J, et al. (2007) Use of a constitutively active hypoxia-inducible factor-1alpha transgene as a therapeutic strategy in no-option critical limb ischemia patients: phase I dose-escalation experience. Circulation 115: 1234-1243.

2. Shigematsu H, Yasuda K, Iwai T, Sasajima T, Ishimaru S, et al. (2010) Randomized, double-blind, placebo-controlled clinical trial of hepatocyte growth factor plasmid for critical limb ischemia. Gene Ther 17: 1152-1161.

3. Powell RJ, Simons M, Mendelsohn FO, Daniel G, Henry TD, et al. (2008) Results of a double-blind, placebo-controlled study to assess the safety of intramuscular injection of hepatocyte growth factor plasmid to improve limb perfusion in patients with critical limb ischemia. Circulation 118: 58-65.

4. Simons M (2005) Angiogenesis: where do we stand now? Circulation 111: 1556-1566.

5. Tongers J, Roncalli JG, Losordo DW (2008) Therapeutic angiogenesis for critical limb ischemia: microvascular therapies coming of age. Circulation 118: 9-16.
6. Ahmed Z, Bicknell R (2009) Angiogenic signalling pathways. Methods Mol Biol 467: 3-24.

7. Donndorf P, Kundt G, Kaminski A, Yerebakan C, Liebold A, et al. (2011) Intramyocardial bone marrow stem cell transplantation during coronary artery bypass surgery: A meta-analysis. J Thorac Cardiovasc Surg 142: 911-920.

8. Fadini GP, Agostini C, Avogaro A (2010) Autologous stem cell therapy for peripheral arterial disease meta-analysis and systematic review of the literature. Atherosclerosis 209:10-17.

9. Madonna R, De CR (2011) Stem cells and growth factor delivery systems for cardiovascular disease. J Biotechnol 154: 291-297

10. Napoli C, Hayashi T, Cacciatore F, Casamassimi A, Casini C, et al. (2011) Endothelial progenitor cells as therapeutic agents in the microcirculation: an update. Atherosclerosis 215: 9-22.

11. Perin EC, Silva GV (2011) Cell-based therapy for chronic ischemic heart disease--a clinical perspective. Cardiovasc Ther 29: 211-217.

12. Povsic TJ, O'Connor CM (2010) Cell therapy for heart failure: the need for a new therapeutic strategy. Expert Rev Cardiovasc Ther 8: 1107-1126.

13. Quyyumi AA, Murrow J (2010) Stem cells in cardiovascular disease. Med Arh 64: $289-294$

14. Storms RW, Trujillo AP, Springer JB, Shah L, Colvin OM, et al. (1999) Isolation of primitive human hematopoietic progenitors on the basis of aldehyde dehydrogenase activity. Proc Natl Acad Sci U S A 96: 9118-9123.

15. Gentry T, Deibert E, Foster SJ, Haley R, Kurtzberg J, et al. (2007) Isolation of early hematopoietic cells, including megakaryocyte progenitors, in the ALDHbright cell population of cryopreserved, banked UC blood. Cytotherapy 9: 569576

16. Gentry T, Foster S, Winstead L, DeibertE, Fiordalisi M, et al. (2007) Simultaneous isolation of human BM hematopoietic, endothelial and mesenchymal progenitor cells by flow sorting based on aldehyde dehydrogenase activity: implications for cell therapy. Cytotherapy 9: 259-274.

17. Capoccia BJ, Robson DL, Levac KD, Maxwell DJ, Hohm SA, et al. (2009) Revascularization of ischemic limbs after transplantation of human bone marrow cells with high aldehyde dehydrogenase activity. Blood 113: 53405351.

18. Balber AE (2011) Concise review: aldehyde dehydrogenase bright stem and progenitor cell populations from normal tissues: characteristics, activities, and emerging uses in regenerative medicine. Stem Cells 29: 570-575.

19. Perin EC, Silva G, Gahremanpour A, Canales J, Zheng Y, et al. (2011) A randomized, controlled study of autologous therapy with bone marrow-derived aldehyde dehydrogenase bright cells in patients with critical limb ischemia Catheter Cardiovasc Interv doi: 10:1002/ccd.23066.

20. Perin EC, Silva GV, Zheng Y, Gahremanpour A, Canales J, et al. (2011) First randomized, controlled double-blind trial of transendocardial injection of autologous aldehyde dehydrogenase-bright stem cells. Am. Heart J 6: 10781087.

21. Sondergaard CS, Hess DA, Maxwell DJ, Weinheimer C, Rosova I, et al. (2010) Human cord blood progenitors with high aldehyde dehydrogenase activity improve vascular density in a model of acute myocardial infarction. J Trans Med 8: 24-37.

22. Bell GI, Broughton HC, Levac KD, Allan DA, Xenocostas A et al. (2011) Transplanted human bone marrow progenitor subtypes stimulate endogenous islet regeneration and revascularization. Stem Cells Dev doi:10.1089/ scd.2010.0583.

23. Marchitti SA, Brocker C, Stagos D, Vasiliou V (2008) Non-P450 aldehyde oxidizing enzymes: the aldehyde dehydrogenase superfamily. Expert Opin Drug Metab Toxicol 4: 697-720.

24. Bleiziffer O, Horch RE, Hammon M, Arkudas A, Naschberger E, et al. (2009) T17b murine embryonal endothelial progenitor cells can be induced towards both proliferation and differentiation in a fibrin matrix. J Cell Mol Med 13: 926 935

25. Camacho M, Rodriguez C, Salazar J, Martinez-Gonzalez J, Ribalta J, et al (2008) Retinoic acid induces PGI synthase expression in human endothelial cells. J Lipid Res 49: 1707-1714. 
Citation: White HS, Smith L, Gentry T, Balber AE (2011) Mechanisms of Action of Human Aldehyde Dehydrogenase Bright Cells in Therapy of Cardiovascular Diseases: Expression Analysis of Angiogenic Factors and Aldehyde Dehydrogenase Isozymes. J Stem Cell Res Ther S1:001. doi:10.4172/2157-7633.S1-001

Page 9 of 9

26. Gaetano C, Catalano A, Illi B, Felici A, Minucci S, et al. (2001) Retinoids induce fibroblast growth factor-2 production in endothelial cells via retinoic acid receptor alpha activation and stimulate angiogenesis in vitro and in vivo. Circ Res 88: E38-E47.

27. Prahalad P, Dakshanamurthy S, Ressom H, Byers SW (2010) Retinoic acid mediates regulation of network formation by COUP-TFII and VE-cadherin expression by TGFbeta receptor kinase in breast cancer cells. PLoS One 5 : e10023.

28. Saito A, Sugawara A, Uruno A, Kudo M, Kagechika H, et al. (2007) All-trans retinoic acid induces in vitro angiogenesis via retinoic acid receptor: possible involvement of paracrine effects of endogenous vascular endothelial growth factor signaling. Endocrinology 148: 1412-1423.

29. Sidell N, Feng Y, Hao L, Wu J, Yu J, et al. (2010) Retinoic acid is a cofactor for translational regulation of vascular endothelial growth factor in human endometrial stromal cells. Mol Endocrinol 24: 148-160.

30. Wu J, Hansen JM, Hao L, Taylor RN, Sidell N (2011) Retinoic acid stimulation of VEGF secretion from human endometrial stromal cells is mediated by production of reactive oxygen species. J Physiol 589: 863-875.

31. Siddikuzzaman, Guruvayoorappan C, Berlin GV (2011) All trans retinoic acid and cancer. Immunopharmacol Immunotoxicol 33: 241-249.

32. Hoffmann S, Rockenstein A, Ramaswamy A, Celik I, Wunderlich A, et al. (2007) Retinoic acid inhibits angiogenesis and tumor growth of thyroid cancer cells. Mol Cell Endocrinol 264: 74-81.

33. Sogno I, Vene R, Sapienza C, Ferrari N, Tosetti F, et al. (2009) Anti-angiogenic properties of chemopreventive drugs: fenretinide as a prototype. Recent Results Cancer Res 181: 71-76.

34. Dutta A, Sen T, Chatterjee (2010) A All-trans retinoic acid (ATRA) downregulates MMP-9 by modulating its regulatory molecules. Cell Adh Migr 4: 409-418.

35. Enciso JM, Konecny CM, Karpen HE, Hirschi KK (2010) Endothelial cell migration during murine yolk sac vascular remodeling occurs by means of a Rac1 and FAK activation pathway in vivo. Dev Dyn 239: 2570-2583.

36. Guruvayoorappan C, Kuttan G (2008) 13 cis-retinoic acid regulates cytokine production and inhibits angiogenesis by disrupting endothelial cell migration and tube formation. J Exp Ther Oncol 7: 173-182.

37. Lai L, Bohnsack BL, Niederreither K, Hirschi KK (2003) Retinoic acid regulates endothelial cell proliferation during vasculogenesis. Development 130: 64656474.

38. Azambuja AP, Portillo-Sanchez V, Rodrigues MV, Omae SV, Schechtman D, et al. (2010) Retinoic acid and VEGF delay smooth muscle relative to endothelial differentiation to coordinate inner and outer coronary vessel wall morphogenesis. Circ Res 107: 204-216.

39. Suzuki Y, Komi Y, Ashino H, Yamashita J, Inoue J, et al. (2004) Retinoic acid controls blood vessel formation by modulating endothelial and mural cell interaction via suppression of Tie2 signaling in vascular progenitor cells. Blood 104: 166-169.

40. Chen K, Bai H, Arzigian M, Gao YX, Bao J, et al. (2010) Endothelial cells regulate cardiomyocyte development from embryonic stem cells. J Cell Biochem 111: 29-39.

41. Sobreira TJ, Marletaz F, Simoes-Costa M, Schechtman D, Pereira AC, et al (2011) Structural shifts of aldehyde dehydrogenase enzymes were instrumental for the early evolution of retinoid-dependent axial patterning in metazoans. Proc Natl Acad Sci U S A 108: 226-231.

42. Rameshwar P, Qiu H, Vatner SF (2010) Stem cells in cardiac repair in an inflammatory microenvironment. Minerva Cardioangiol 58: 127-146.

43. Mohyeldin A, Garzon-Muvdi T, Quinones-Hinojosa A (2010) Oxygen in stem cell biology: a critical component of the stem cell niche. Cell Stem Cell 7: 150161

44. Muramoto GG, Russell JL, Safi R, Salter AB, Himburg HA, et al. (2010) Inhibition of aldehyde dehydrogenase expands hematopoietic stem cells with radioprotective capacity. Stem Cells 28: 523-534.

45. Marcato P, Dean CA, Pan D, Araslanova R, Gillis M, et al. (2011) Aldehyde dehydrogenase activity of breast cancer stem cells is primarily due to isoform ALDH1A3 and its expression is predictive of metastasis. Stem Cells 29: 32-45.

46. Mantel C, Messina-Graham SV, Broxmeyer HE (2011) Superoxide flashes, reactive oxygen species, and the mitochondrial permeability transition pore: potential implications for hematopoietic stem cell function. Curr Opin Hemato 18: $208-213$.

47. Mantel C, Messina-Graham S, Broxmeyer HE (2011) Upregulation of nascent mitochondrial biogenesis in mouse hematopoietic stem cells parallels upregulation of CD34 and loss of pluripotency: a potential strategy for reducing oxidative risk in stem cells. Cell Cycle 9: 2008-2017.

48. Van der Pouw Kraan TC, Schirmer SH, Fledderus JO, Moerland PD, Baggen JM, et al. (2010) Expression of a retinoic acid signature in circulating CD34 cells from coronary artery disease patients. BMC Genomics 11: 388.

49. Bernabeu C, Conley BA, Vary CP (2007) Novel biochemical pathways of endoglin in vascular cell physiology. J Cell Biochem 102: 1375-1388.

50. Dallas NA, Samuel S, Xia L, Fan F, Gray MJ, et al. (2008) Endoglin (CD105): a marker of tumor vasculature and potential target for therapy. Clin Cancer Res 14: 1931-1937

51. Arvanitis D, Davy A (2008) Eph/ephrin signaling: networks. Genes Dev 22: 416 429

52. Kuijper S, Turner CJ, Adams RH (2007) Regulation of angiogenesis by Ephephrin interactions. Trends Cardiovasc Med 17: 145-151.

53. Pitulescu ME, Adams RH (2010) Eph/ephrin molecules--a hub for signaling and endocytosis. Genes Dev 24: 2480-2492. 